\section{LEPTIN LEVELS AND BODY COMPOSITION DURING THE FIRST YEAR OF LIFE \\ IN PREMATURE INFANTS AND THEIR RELATIONSHIP WITH NUTRITION}

N.M. Díaz-Gómez ${ }^{1}$, E. Doménech ${ }^{2}$, F. Barroso ${ }^{3}$, C. Cortabarría ${ }^{4}$

${ }^{1}$ Department of Nursing, Universidad de La Laguna, ${ }^{2}$ Department of Pediatrics, Hospital Universitario de Canarias. Universidad de La Laguna, ${ }^{3}$ Department of Pediatrics, Universidad de La Laguna, ${ }^{4}$ Department of Pediatrics, Hospital Universitario de Canarias, La Laguna, Spain

Aims: To analyze the changes in leptin serum levels (LSL), body mass index (BMI) and total body fat (TBF) during the first year in preterm infants (PI), comparing those who received an adapted standard formula (AF) after hospital discharge with those fed with a formula for premature infants (PF).

Methods: Seventy-two PI were included in the study $(32.1 \pm 1.9$ weeks and 1,712 $\pm 340 \mathrm{~g})$. Thirtysix received AF (Energy: $67 \mathrm{kcal} / 100 \mathrm{ml}$; proteins: $1.2 \mathrm{~g} / 100 \mathrm{ml}$ ) and 36 received PF (Energy: $81 \mathrm{kcal} / 100 \mathrm{ml}$, proteins: $2.3 \mathrm{~g} / 100 \mathrm{ml}$ ) from hospital discharge until corrected postnatal age (CA) of 4 months. The data were obtained at 36 weeks postconceptional age (PCA, baseline), and at 3, 6 and 12 months CA.

Results: In all the PI studied, skinfold measurements and percentage TBF showed an important increase from baseline to 3 months CA, decreasing from 6 to 12 months CA. LSL showed a significant correlation with these variables at 3 and 6 months $C A$, and also with $\mathrm{BMI}$ at 6 months $\mathrm{CA}$.

Throughout the study period, the infants fed with PF showed significant higher values for $\mathrm{BMI}$ at 6 and 12 months $\mathrm{CA}$, as well as for the increase in BMI and sum of 4 skinfolds from 36 weeks PCA to 6 months CA. There were no significant differences between the two groups in size or LSL.

Conclusions: Nutrition with a formula of greater energy and protein content during the first four months after hospital discharge in PI was associated with higher BMI and body fat without affecting statural growth or LSL.

\section{PROBIOTICS SUPPLEMENTATION INCREASES INTESTINAL BLOOD FLOW IN EXTREMELY LOW BIRTH WEIGHT (ELBW) NEONATES}

T. Havranek ${ }^{1}$, M. Al-Hosni ${ }^{1}$, E.S. Armbrecht ${ }^{2}$

${ }^{1}$ Pediatrics/Neonatology, ${ }^{2}$ Center for Outcomes Research (SLUCOR), Saint Louis University, Saint Louis, MO, USA

Background: Proposed benefits of probiotics supplementation are multiple; recently, vasoactive properties of trace amines, produced by probiotics, have been shown to affect mesenteric vasculature in animal models. The effects of probiotics on intestinal blood flow have not yet been described.

Aims: To determine whether probiotics supplementation affects intestinal blood flow velocity in ELBW neonates.

Methods: In this randomized, double-blind, placebo-controlled study probiotics (500 M CFU of Lactobacillus rhamnosus and 500 million CFU of Bifidobacterium infantis) were added to the first enteral feeding and continued until discharge or 34 weeks postmenstrual age. Pulsed Doppler measured preprandial and postprandial (at 30 and $60 \mathrm{~min}$ ) time-averaged mean, peak and end diastolic velocities (TAMV, PSV, EDV) during second week of life after 7 days of probiotics supplementation. Mean values across time were graphed for probiotics and control groups. The percent change from preprandial to 60 min post was compared by t-test, for each measurement method.

Results: Thirty-one infants were studied, 15 randomized to probiotics and 16 to placebo. Postprandial TAMV were increased significantly in probiotics group ( $p=0.048)$, with PSV and EDV showing a trend.

There were no significant differences between groups in mean birth weight, gestational age, the time of the study, volume and type of feedings $(p>0.05)$. The percent of infants undergoing phototherapy, receiving caffeine or being diagnosed with patent ductus arteriosus was similar between groups $(p>0.05)$.

Conclusions: Probiotics administration significantly increases intestinal blood flow in ELBW preterm neonates when compared to placebo group. 